\title{
"L'accouchement c'est la guerre"- De quelques problèmes liés à l'accouchement en milieu rural nigérien
}

Jean-Pierre Olivier de Sardan, Adamou Moumouni et Aboubacar Souley

\section{CpenEdition}

Journals

Édition électronique

URL : https://journals.openedition.org/apad/483

DOI : 10.4000/apad.483

ISSN : 1950-6929

Éditeur

LIT Verlag

Édition imprimée

Date de publication : 1 juin 1999

Référence électronique

Jean-Pierre Olivier de Sardan, Adamou Moumouni et Aboubacar Souley, «"L'accouchement c'est la guerre"- De quelques problèmes liés à l'accouchement en milieu rural nigérien », Bulletin de l'APAD [En ligne], 17| 1999, mis en ligne le 04 octobre 2006, consulté le 21 septembre 2021. URL : http:// journals.openedition.org/apad/483; DOI : https://doi.org/10.4000/apad.483

Ce document a été généré automatiquement le 21 septembre 2021.

Bulletin de I'APAD 


\title{
"L'accouchement c'est la guerre"- De quelques problèmes liés à l'accouchement en milieu rural nigérien
}

\author{
Jean-Pierre Olivier de Sardan, Adamou Moumouni et Aboubacar Souley
}

"L'accouchement, c'est comme une bataille, c'est comme une guerre ; toute femme qui, ayant été enceinte, et ayant accouché, ne s'agenouille pas pour rendre grâce à Dieu, ne sera plus jamais une croyante" (entretien avec des femmes de Koutoukallé) Introduction

1 On sait que la mortalité maternelle est forte en Afrique, et particulièrement au Niger (autour de $10 \%$ ) ${ }^{1}$. Les projets "Maternité sans risques" ont du pain sur la planche, tant en milieu rural qu'en milieu urbain. Les conditions sont d'ailleurs très différentes dans ces deux environnements. L'accouchement en maternité est dominant à Niamey (mais non exclusif, puisque $26 \%$ des femmes y accouchent encore à domicile ${ }^{2}$ ) et l'évacuation relativement facile vers la maternité centrale en cas de complications. Par contre, dans les campagnes, l'accouchement à domicile domine largement ${ }^{3}$, complété par des accouchements dans des centres de santé ruraux (dispensaires) là où il en existe ${ }^{4}$, sous la responsabilité d'un simple infirmier(ère) - les sages-femmes sont concentrées dans la capitale, et, sinon, dans quelques villes secondaires ${ }^{5}$-, avec d'énormes problèmes d'évacuation.

2 Ce texte essentiellement descriptif concerne uniquement l'accouchement et ses problèmes en milieu rural ${ }^{6}$. L'enquête a été menée pendant 3 mois dans 12 villages nigériens, dans l'arrondissement de Birni, en zone zarmaphone et dans l'arrondissement de Mayahi, en zone hausaphone. Il faut noter que c'est la première fois qu'une étude comparative qualitative de ce type est menée simultanément dans les deux principales langues et cultures nigériennes (un élargissement aux autres langues et cultures serait évidemment souhaitable). Elle fait d'ailleurs ressortir une grande convergence des pratiques et représentations populaires. Après l'évocation d'un cas d'accouchement à problème, nous décrirons successivement l'accouchement 
"traditionnel" à la maison et l'attitude face aux complications, puis l'accouchement au dispensaire, avant d'analyser plus en détail le rôle des matrones (dans la mesure où celles-ci ont fait l'objet de nombreux programmes de formation dans le cadre des "soins de santé primaire"), puis la question, centrale en matière de santé publique, des évacuations en milieu rural.

\section{L'accouchement de Nana}

Nana habite à Mashe Jan Baushi. Elle n'a jamais été à l'école. C'est sa première grossesse. La grossesse s'est passée sans difficulté aucune. Nana a suivi régulièrement les consultations prénatales $(\mathrm{CPN})$ au Centre de soins intégrés (CSI) de Dan Mairo, situé à environ $15 \mathrm{~km}$ de son village. Elle soutient qu'elle s'est rendue plus de quatre fois à Dan Mairo pour les CPN. Le trajet se faisait à pied. contentée de la nivaquine, achetée au CSI à l'occasion des CPN. Selon elle, c'est un traitement préventif contre la fièvre conseillé par les infirmiers.

Lorsque le travail a commencé, elle n'a rien dit à personne. C'était le soir, et elle a passé toute la nuit en travail. Son mari était absent, parti en exode, au moment des faits. Mais elle n'a prévenu ni sa mère, ni son beau-père. Le travail était intermittent. Au petit matin, Nana a déclaré qu'elle souffrait de douleurs au bas-ventre. Alertée, la grand-mère de son mari lui a donné une décoction à boire. Nana dit tout ignorer de la nature et des effets de cette décoction: "Comment pourrai-je savoir? Je n'en sais rien. Ce sont eux qui savent ce que c'est. Comment diable vais-je oser poser de telles questions ?". Quant au beau-père de Nana, il lui a fait boire "le nom de Dieu" (sunan Alla en hausa, appelé aussi rubutu, c'est-à-dire l'eau avec laquelle on a lavé une tablette sur laquelle étaient tracés à l'encre des versets coraniques). Le beau-père s'en explique: "Tu sais c'est une pratique courante, parfois, si les choses n'évoluent pas comme on le souhaite, on a recours au rubutu afin qu'Alla nous aide". Marabout, l'homme a lui-même prescrit et fait administrer le rubutu.

Ces produits ne firent, manifestement, aucun effet. Le travail durait toujours, sans qu'il y ait progrès. Le beau-père de Nana proposa, alors, de la conduire au CSI, à Dan Mairo. Le soleil était haut sur l'horizon. Il semblerait que les femmes (essentiellement les "vieilles", sans qu'on puisse dire exactement qui) n'aient pas été d'accord dans un premier temps. Le beau-père reconnaît que des gens se sont opposés à son idée quand il l'a émise la toute première fois, mais il n'a pas voulu dire qui. De même, la grand-mère de Nana affirme qu'elle ne se rappelle pas de cet épisode ${ }^{7}$.

En fait, depuis le matin, c'était l'affluence autour de Nana. Chacun y allait de son commentaire, de ses conseils et, parfois, de ses contributions thérapeutiques. Il faut noter, en règle général, que les vieilles personnes voient d'un mauvais œil l'accouchement à publicité, et notamment l'accouchement au CSI. On dit, dans ce cas, que la femme n'a pas accouché seule, d'elle même, qu'elle s'est fait aider, que c'est l'infirmier qui l'a fait accoucher. On distinguerait, ainsi, l'accouchement normal, travail de la femme non assistée, souhaité et source de considération, et l'accouchement dit "du docteur" haihuwar likita). au CSI, non souhaité et quelque peu "honteux".

Finalement, après la dernière prière, la nuit étant déjà tombée, après 24 heures de travail et de douleurs, le beau-père trancha et décida qu'il fallait évacuer Nana. En l'absence de son fils, c'est lui qui avait autorité sur la femme de ce dernier. Il était le seul homme à la maison.

L'évacuation a eu lieu en pleine nuit: "je pense qu'au moment où nous sommes partis personne n'était éveillé". Elle s'est faite sur une charrette asine. Outre Nana, il y avait son beau-père, un ami de ce dernier, la grand-mère de Nana et celle de son mari, la mère de Nana et l'une des tantes paternelles de son mari. Le trajet, de Mashe à Dan Mairo, dura entre 3 et 4 heures selon les versions. Il y eut plusieurs 
arrêts, selon Nana, à sa demande, soit pour qu'elle urine, soit quand elle souffrait trop. Sa mère et la grand-mère de son mari soutiennent par contre qu'il n'y a eu aucun arrêt sur le chemin. De même le beau-père affirme: "Dès que nous sommes partis, nous ne nous sommes arrêtés qu'une fois arrivés à Dan Mairo ; d'ailleurs moi je ne faisais que faire galoper l'âne".

L'accouchement a eu lieu au CSI de Dan Mairo vers 9 heures du matin. Il semble que tout s'est bien passé. La poche des eaux a éclaté peu avant la délivrance. Le placenta a presque immédiatement suivi. Avant l'accouchement, l'infirmier avait administré des ocytociques à la parturiente. Pour le remercier, le beau-père lui a donné 300 francs, en plus du don quasi conventionnel d'un morceau de savon de Marseille et d'une somme de 200 francs. Quand la jeune maman revint, après quelques jours, pour la pesée de l'enfant, elle apporta quelques mesures de mil, des condiments et un peu d'argent (elle n'a pas voulu dire combien) à l'infirmier.

Juste après l'accouchement, Nana dit que l'infirmier a exigé qu'on mette le bébé au sein. Ce qui fut fait. Cependant, dès son retour à Mashe, quelques heures plus tard, les "vieilles" exigèrent de procéder au "test du lait". On recueillit quelques gouttes du lait de la jeune maman dans un bol; on fit chauffer un couteau dans des braises; quand il devint tout rouge, on versa dessus le lait de la jeune maman. Le lait colla au couteau, ce qui était indicatif de la "maladie du lait" appelé kaikai (littéralement démangeaisons). Aussi a-t-on proscrit le sein au nouveau-né durant trois jours (trois jours parce que c'était un garçon; pour une fille cela aurait été quatre jours), période pendant laquelle on lui a fait boire du lait de chèvre légèrement réchauffé, et la décoction fortifiante et préventive dauri (littéralement "amer") administrée à tous les jeunes enfants, tandis que sa mère prenait une potion "anti kaikai".

Travail et accouchement : représentations et pratiques populaires

De l'avis général, le début du travail (en zarma hayzaNay, et en hausa nakuda) se manifeste toujours par des douleurs au bas-ventre et souvent par des douleurs au dos. Il $\mathrm{y}$ a des cas où le travail se signale par l'écoulement de sang

"II y a des gens qui disent qu'elles font le travail avec du sang"

L'éclatement de la poche des eaux signale la phase terminale du travail.

"Les Zarma disent que si la poche des eaux s'éclate, il ne reste plus qu'à accoucher"

6 Toutefois, on observe parfois un décalage.

"La poche des eaux peut s'éclater sans que l'accouchement n'intervienne vite"

7 Par ailleurs, on attribue un effet de régulateur thermique à la poche des eaux. L'éclatement de la poche des eaux entraînerait un refroidissement du corps.

"Dès que la poche des eaux est éclatée, ton corps devient frais car c'est la poche des

eaux qui fait chauffer le corps"

8 Traditionnellement, les accoucheuses n'intervenaient jamais pour percer la poche des eaux, cela doit s'opérer naturellement. On pense aussi que certaines femmes "vomissent" la poche des eaux ${ }^{8}$.

"Il y a des femmes qui vomissent la poche des eaux"

La durée normale du travail n'excède pas un jour même si certaines femmes parlent de plus.

"Moi que tu vois, j'ai fait trois jours de travail pourtant cela ne m'a rien fait"

10 L'opinion dominante est que la durée du travail est liée aux particularités de chaque enfant (cf. en zarma le dicton "tout enfant a sa propre façon de marcher", zanka kul nda nga ce dira). Douleur et durée varient beaucoup. Un travail qui dure longtemps est appelé en hausa nauyin guywa, c'est-à-dire "genoux lourds". Il peut être dû par exemple à un enfant trop volumineux. 
"-Qu'est-ce qui fait le nauyin guywa? c'est si par exemple l'enfant est robuste, il est gros"

"C'est la plus grande douleur, il n'y a pas une douleur aussi grande, parmi toutes les douleurs. Ce que tu ressens au moment du travail est plus douloureux que toute douleur que tu connais. Parfois ça dure un jour ou deux jours. Certaines femmes vont jusqu'à une semaine. D'autres, par contre, dès que ça commence, elles accouchent. Ca dépend des femmes. Il y en a qui ont un "bon genou". Il y en a d'autres qui traînent le travail pendant longtemps, et souffrent avant d'être délivrée"

Plus le travail dure, plus il devient source de craintes.

"Si on voit que le travail dure, ça commence à inquiéter les gens, on va chercher un secours"

On impute parfois la responsabilité d'un travail trop long à weyno (ainsi qu'à moosey, jaunisse), en zarma, ou à zaki, en hausa ${ }^{9}$.

"Celles qui souffrent de weyno doivent nécessairement le faire sortir par les urines ou par les selles. C'est ce qui explique le fait d'uriner ou d'aller aux selles à chaque instant"

Le weyno ou le zaki peut provoquer également une sorte de "faux travail".

"Lorsque l'enfant bouge un peu, tu as l'impression qu'il va sortir, or il n'en rien c'est plutôt le weyno"

"Weyno soulève l'enfant comme si c'est le travail alors que ce n'est pas cela"

On parle parfois d'un "travail du septième mois, qui marquerait le renversement (bare en zarma, jirkice en hausa) de l'enfant, et qui préfigurerait un accouchement sans problèmes.

"Certaines femmes ressentent le travail dès le septième mois. Si tu ressens le travail dès le septième mois, tu accoucheras facilement, sans grosses difficultés. Tu sais, c'est le moment du renversement du bébé, sa tête se renverse. Mais ce n'est pas le travail de l'accouchement. Ça ne veut pas dire que la femme va accoucher alors".

Pour prévenir un travail long, les parturientes, vers la fin de leur grossesse, peuvent boire des décoctions censées accélérer le travail (c'est, en pays hausa, systématique pour les primipares, fréquent chez les multipares). Elles peuvent aussi avoir recours aux marabouts, ou encore aux prêtres des génies ou aux guérisseurs (zimma, boka). On trouve ici ou là d'autres procédés encore, comme cette habitude, presque abandonnée aujourd'hui, de faire boire à la femme une eau dans laquelle la ceinture du pantalon traditionnel de son mari aura macéré... En cas d'accouchement difficile, les accoucheuses en milieu zarma utilisaient un zumandi tira (charme pour faciliter l'accouchement) qu'on nouait aux cheveux de la parturiente. Ce charme avait deux origines : il était confectionné localement par des marabouts spécialistes ou bien il était acheté auprès de commerçants ambulants provenant du pays hausa.

Pour bon nombre de femmes, l'idéal est d'accoucher dans la discrétion, c'est-à. dire sans l'assistance de la matrone ou de l'accoucheuse. Un tel acte est valorisé socialement et considéré comme une sorte de bravoure. Une telle femme sera citée en référence par ses amies. La solitude est aussi une question de pudeur.

"Pour l'accouchement, ce qu'on veut, c'est que tu le fasses seule, sans l'assistance de personne. Comme personne ne t'a entendu, personne n'ira raconter"

Ainsi, en général, les accouchements à domicile se font avant l'arrivée de la matrone ou de l'accoucheuse, qui n'est appelée que pour couper le cordon. 

Elle aurait en effet des vertus antiseptiques, adoucissantes et cicatrisantes. On dit qu'alors il faut "chasser le mauvais sang". D'où l'eau chaude, les massages abdominaux, les décoctions. Le ventre est aussi bandé de façon serrée, ce qui doit permettre également qu'il puisse reprendre sa forme normale. La nouvelle accouchée (antuga en zarma, mai biki en hausa), qui ne sort pas, est complètement prise en charge, pour sa toilette comme pour les autres tâches, au moins pendant les sept jours qui séparent l'accouchement du baptême. En général une parente est attachée particulièrement à son service (antuga batukow en zarma, mai hokon biki en hausa). Diverses prescriptions ou interdits, variables selon les contextes locaux, accompagnent ces sept jours, voire perdurent jusqu'au "quarantième jour", qui marque la fin de la période de l'accouchement (par exemple: ne pas balayer, ou ne pas porter certains objets; verser, pour dissuader les mauvais génies, trois ou quatre tas de cendre à un croisement 3 ou 4 jours après l'accouchement - trois, pour un garçon, quatre pour une fille etc.).

21 Certains enfants naissent dans une membrane (foolo, litt. sac, enveloppe, en zarma). On les appelle mai riga en hausa (litt. possesseur d'une chemise), terme repris en zarma.

"Il y a des cas où tu vois une sorte de corde qui sort, dès que tu vois ça et que tu vois l'enfant sortir fais vite pour déchirer, car c'est l'accouchement dans l'enveloppe. Quand tu ignores cela, l'enfant se débat, mais tu ne sais pas ce que c'est, l'enfant peut mourir. C'est au dispensaire qu'on nous a appris ça"

D'autres naissent inanimés. En ce cas on ne procédait à aucune tentative de réanimation.

"Avant, si l'enfant ne respirait pas, on pensait qu'il était mort, on l'enterrait"

Pour un accouchement faisant suite à une série d'accouchements d'enfants mort-nés ou morts dans les premiers jours, on tentait de conjurer le mauvais sort, en pratiquant l'accouchement dans des sites dévalorisés, affichant ainsi le peu de valeur de l'enfant (pour dissuader génies ou sorciers de s'y intéresser et de le prendre) : ainsi, en zarma, l'écurie (kangey), l'endroit où se tient l'âne (binji), le poulailler (fiti). On lavera l'enfant avec un morceau de canari ( $\mathrm{cambu}$ ) servant à abreuver les volailles. Ces pratiques tombent peu à peu en désuétude, on en trouve surtout trace dans les surnoms donnés à ce type d'enfants (en zarma: Cambu, Kangey; en hausa: Abarshi, "laissez-le", Abarka, "laissez-la"). 
24 En ce qui concerne les prématurés l'opinion générale est que ceux du $7^{\text {ème }}$ mois vont survivre ${ }^{11}$, mais pas ceux du $8^{\text {ème }}$ mois. Les prématurés étaient autrefois placé dans une sorte de "couscoussier" (appelé en zarma dambu) et ainsi chauffés sur un feu de tiges de mil. En pays hausa le procédé était différent.

"Nous, nous ne mettons pas le nouveau-né du $7^{\text {ème }}$ mois dans un couscoussier. Ici, si tu enfantes au septième mois, si Dieu fait vivre l'enfant, nous prenons du bagaruwa (plante), nous le plongeons dans l'eau et le faisons décanter. Après avoir lavé l'enfant de ses saletés, nous le lavons avec le bagaruwa, à intervalles réguliers, pendant une semaine"

Aussitôt après l'expulsion, la première tâche consiste à couper le cordon ombilical. La coupure du cordon se fait aujourd'hui avec une lame de rasoir, autrefois avec un morceau de tige de mil.

"Dans le passé, on coupait à l'aide de quatre tiges pour la fille et trois tiges pour le garçon. On évalue les points de raccordement pour couper : si c'est un garçon on coupe au troisième, chez la fille on coupe au quatrième"

La cautérisation du cordon se fait avec un couteau ou un morceau de poterie chauffé au rouge.

Le placenta

Le placenta est extrêmement important. En un sens on s'occupe d'abord de lui, avant même de s'occuper du nouveau-né. On le lave et on l'enterre selon des modalités précises.

"C'est le placenta dont on s'occupe d'abord, avant de laver le bébé. Quand on coupe le cordon ombilical, on lave le placenta, on lui creuse un trou. Le trou doit être profond jusqu'au coude. Nous lui faisons un nid. Le trou du placenta, si c'est un garçon c'est au nord sur la droite qu'on creuse le trou. Si c'est une fille c'est sur la gauche. Nous demandons des restes de canaris cassés et du crottin de chèvre. Après avoir placé le placenta nous posons les morceaux de canari cassé, ensuite nous mettons les crottins de chèvre, après quoi nous refermons le trou. Puis quelqu'un verse de l'eau. Si c'est un garçon, on verse 3 fois, si c'est une fille on verse 4 fois. On lave ensuite le bébé, puis nous ramassons le sang. Ensuite on nous donne une bouilloire, nous faisons les ablutions à l'enfant, les mêmes que nous faisons pour prier. Nous lui faisons un appel de prière à l'oreille. Alors nous le posons sur le lit. Quant au sang, on le ramasse et il y a un trou pour ça, on le verse dedans. C'est avec nos mains qu'on le ramasse sur le lieu de l'accouchement, on verse de l'eau, on lave par terre. Avec un balai, nous recouvrons l'endroit de sable. Depuis l'origine du monde c'est ainsi qu'on procède"

"Le placenta est mis dans un récipient, on le pose dans le trou en prenant soin de placer par le haut car si on le renverse, la femme n'accouchera plus"

Le placenta est appelé en zarma cora (litt. ami) ou hangasin (litt. compagnon) et en hausa ma'haihwa (litt. la chose avec laquelle on accouche), saraunya (litt. reine) ou uwa (litt. mère). On ne proclame jamais publiquement la naissance d'un enfant tant que le placenta n'est pas lui aussi sorti. L'enfant et le placenta peuvent "tomber" quasi simultanément, tout comme le placenta peut prendre un certain temps avant de "tomber" : on dit alors que l"'ami" ou la "reine" se repose. S'il refuse de venir, on utilise les procédés suivants :

- on masse le ventre de la parturiente tout en récitant des versets coraniques

- on introduit un bâton dans sa gorge pour la faire tousser

- Toujours pour la faire tousser, on lui fait respirer la fumée d'un feu où l'on a mis du piment.

- on tente de la faire éternuer en lui faisant priser du tabac

- on donne une tape à l'enfant afin qu'il pousse un cri, pour "appeler" son "compagnon" 
- on prépare un breuvage rituel que l'on place dans une calebasse ; la femme doit le boire en tenant la calebasse entre ses dents ; sitôt après avoir bu elle doit lâcher la calebasse afin qu'elle tombe renversée : le placenta doit alors tomber.

- on attache une pierre au cordon (on peut aussi attacher le cordon au pied de la mère, afin que le placenta ne "remonte" pas : ceci est fait lors d'évacuations en charrette vers un dispensaire pour une rétention placentaire rebelle aux méthodes "traditionnelles")

On évoque aussi un passé révolu où certains éleveurs, ayant une longue expérience avec leurs animaux, étaient parfois appelés pour les femmes en difficulté. Dans chaque village, aujourd'hui, il y a des marabouts (et parfois des zimma ou des boka) qui sont réputés pour leurs prières ou leurs talismans en cas de rétention placentaire. Ils sont aujourd'hui le seul recours local (l'évacuation vers un dispensaire, quand elle est possible, étant désormais courante ; cf. ci-dessous).

C'est à eux aussi que l'on fait appel pour les autres risques de l'accouchement, qui, outre la rétention placentaire et certaines complications post-partum (cf. ci-dessous) sont essentiellement, aux yeux des femmes, le travail "trop long", et les mauvaises présentations. Il peut y avoir le fait que l'enfant se "mette en travers" (garu, en zarma), ou qu'il se présente "par le dos" (c'est-à-dire par le siège), ou que la tête soit mal placée. Mais c'est la présentation par les pieds, appelée "sauter-atterrir" (en zarma jippoyan, en hausa ya diro) qui est la plus crainte.

"La tête s'est présentée la première, mais de mauvaise façon, car on ne peut avoir un accouchement facile si elle se présente comme cela"

"Il y a des accouchements où ce sont les pieds qui sortent en premier. On les appelle "sauter" (jippo). Parfois la femme en couches peut en mourir"

"Les enfants qui tombent par les pieds, c'est l'accouchement appelé bari zumandi (litt. "faire descendre comme un cheval") ${ }^{12}$, ce n'est pas une position normale, la mère ou l'enfant peuvent mourir. Le bari zumandi, c'est une bataille, c'est pourquoi les gens n'aiment pas en parler"

Une mauvaise présentation peut, en particulier, être due au fait que l'enfant va "remonter" vers l'estomac ${ }^{13}$. Aussi, si l'on pense que tel est le cas, va-t-on ligaturer la poitrine avec un pagne afin d'éviter cette montée et de ramener l'enfant vers le bas.

"Autrefois, peu avant l'accouchement, on te ceignait avec un pagne la partie comprise entre le cœur et le ventre, on l'attachait fort pour que l'enfant ne change pas de position, et que la mère puisse s'agenouiller pour accoucher, sinon l'enfant pourrait remonter et ce serait dangereux".

En cas de travail trop long (le début du travail étant, pour les femmes, le moment des premières douleurs, ce qui, d'un point de vue gynécologique, inclut le "faux travail") on fait parfois absorber à la parturiente une décoction, qui peut être la même qui est utilisée en d'autres circonstances comme abortif.

"Pour faire sortir l'enfant, je donne une décoction à base de bantan banda (plante), ou bien un breuvage magique"

En cas de mauvaise présentation, on ne procède qu'à des massages, jamais à des tractions ou des manœuvres internes.

Des convulsions éventuelles seront imputées à un génie, ou à l'épilepsie (circira en zarma, borin jaki en hausa). L'éclampsie n'est pas connue (sauf là où des séances d'éducation pour la santé ont popularisé l'expression hausa nouvelle de borin gishiri, la "transe du sel").

"Avant-hier, il y a eu une femme qui a eu une crise d'épilepsie pendant le travail,

l'accouchement a été difficile, les parents nous ont dit que jamais elle n'a eu des 
choses pareilles, nous nous sommes dit que c'est l'épilepsie, la crise s'est déclenchée quand elle est montée sur la table d'accouchement" (c'est une infirmière qui parle...)

Parfois on peut imputer certaines difficultés de l'accouchement à la transgression d'interdits. De même "sorciers mangeurs d'âmes" et génies malfaisants peuvent intervenir négativement, et en particulier effrayer la parturiente. Même le génie protecteur de la famille, si on lui manque d'égards, peut susciter des difficultés.

"- Est-ce qu'un sorcier (nya-wa-koy) peut causer des difficultés d'accouchement? Certes, on ne veut pas du tout qu'il y en ait un de présent là où quelqu'un accouche!"

"Pour certaines personnes c'est le "génie de la maison" (fu kali en zarma) qui est à la base des problèmes. C'est pourquoi, quand la grossesse touche à son terme, on fait des présents au génie de la maison, un poulet, ou une étoffe"

Mais le plus souvent les complications relèvent pour les gens du destin ou de la fatalité, autrement dit de Dieu. En fait, face aux dangers de l'accouchement, et à la mort toujours possible, les femmes et les hommes se sentent très largement démunis. Il n'y a guère d'autre recours que Dieu, d'où cet appel systématique aux marabouts.

"Lorsqu'il y a des difficultés, les gens ne vont nulle part ailleurs que chez les marabouts"

"Les Zarma disent que la femme enceinte à un pied dans l'au-delà, un pied dans le monde d'ici bas"

Une femme morte en couches (appelée weyhay en zarma ${ }^{14}$ ) était autrefois (et reste encore parfois) perçue comme une "mauvaise morte", susceptible de se manifester sous la forme d'une revenante. Ses jours ont été interrompus en quelque sorte inopinément, trop tôt, et son "double" (biya en zarma) risque de revenir tourmenter les vivants. Aussi $\mathrm{y}$ avait-il des rituels particuliers. En pays songhay, une sorte de caste de fossoyeursmagiciens, les hirow ${ }^{15}$, étaient chargée d'ensevelir les femmes mortes en couches ainsi que les autres décès de personnes frappées subitement dans la force de l'âge. Cette pratique est aujourd'hui abandonnée.

8 Certaines pratiques locales survivent, là ou les populations sont éloignées d'un centre médical, pour quatre types de complications post-partum : la déchirure, l'hémorragie, le "sang accumulé" et les douleurs abdominales aiguës (en général des infections).

La déchirure

9 La déchirure (kottuyan en zarma, kari en hausa) concernerait, dit-on, plutôt les primipares. En l'absence d'un dispensaire, on utilise soit des plantes, soit un procédé visant à cicatriser "à chaud" la déchirure.

Dans le premier cas, on broie dans un mortier des herbes ou des. feuilles. La pâte ainsi obtenue est appliquée sous forme d'emplâtre sur la déchirure. Ceci est censé arrêter le sang qui s'écoule, empêcher toute infection et cicatriser la plaie. Le procédé de cicatrisation "à chaud" est plus traumatisant.

"R. On place un mortier devant elle, elle s'assoit, elle ne se couche pas

Q. Elle s'assoit sur le mortier?

R. Non, on a creusé un trou, on y a amené des braises (qu'on recouvre d'un peu de terre), et elle s'assoit dessus (rires).

Q. Je n'ai pas compris

R. Pas sur le mortier, près du mortier, et on assoit la personne comme ça.

Q. Le mortier est debout?

R. Le mortier est là devant toi, on te place un oreiller pour que tu t'assoies comme ça. 
Q. C'est ce que je dis, le mortier est debout?

R. Oui il est debout, oui! Alors tu t'assois jusqu'à un certain temps (au-dessus des braises). Après on te lève et tu montes sur un lit pour te coucher.

Q. Où met-on le feu?

R. Le feu! On creuse un trou comme ça.

Q. Et alors la personne tient le mortier?

R. Oui elle tient le mortier"

"Si la femme a fait une déchirure, deux jours après l'avoir mise au-dessus des braises, le barbier (wanzami) vient, il lui met un emplâtre"

L'hémorragie décoctions et, parfois, d'emplâtres (contrairement au cas de la déchirure, l'emplâtre, ici, va être placé sur la tête de la femme).

"Dans notre tradition, on cherche une sorte particulière de farine, on mélange, on broie, on trempe, et on donne à boire (? ? ?) , c'est contre le sang"

"On cueille des plantes qu'on pile et on les pose sur sa tête"

Le sang accumulé

Parfois, le sang s'accumule dans le "ventre" au lieu d'être expulsé. On parle alors en zarma de kuri gumyan (sang blotti) et en hausa de ciwon jini (maladie du sang). Pour les gens, ce sang non expulsé rend malade la femme et est susceptible de la tuer. Pour le faire sortir, il y a divers procédés : on fait des massages, on donne à boire de l'eau chaude, on administre une décoction à base de plantes (par exemple masa, en zarma ; daudar baiwa en hausa).

"Ciwon jini, quand la femme accouche, le sang se rassemble, il te noue. Quand il se lève, tu ne fais que gémir, tu ne fais que gémir. On te prépare du savon, tu bois. On te prépare du yodo, tu bois. On te prépare du balma, tu bois. On cueille du daudar baiwa en brousse, on pile, on te prépare, tu bois. Alors il te lâche. Cependant, à présent qu'il y a le centre médical, si on te fait l'injection, tu n'auras pas cette maladie. (...) Sinon, deux jours après (l'accouchement), au troisième jour, après la douche matinale, tu appelles une vieille afin qu'elle te masse les côtes. Elle masse, elle masse et le sang sort, il sort, pour celle qui en a trop. Pour celle chez qui le sang forme des caillots (kasko, litt. morceau de pot cassé) il ne se sépare jamais de toi. $\mathrm{Tu}$ passes la nuit à gémir, mais dès le petit matin, c'est fini. Cependant, après la quarantaine, cela cesse totalement"

Les douleurs abdominales aigües

Des douleurs aigües (en général évocatrices, pour un médecin, d'infections) peuvent se manifester dans le bas-ventre de la femme, pendant les jours qui suivent l'accouchement. On parle alors parfois, en hausa, de naginza. On impute naginza au destin (Dieu), mais aussi aux frottements dus à la sortie du nouveau-né, et, parfois, au manque d'hygiène. La forme "visible" de naginza se caractérise par des écorchures, des petits boutons, souvent blanchâtres, des écoulements visqueux. La forme "invisible", assortie de douleurs insupportables au bas-ventre, peut "attaquer" le cœur et tuer.

"Il y a celui qui te fait une piqûre là-bas à l'intérieur dans le ventre, et il attrape ton cœur. Alors, tu entendras dire que le naginza d'une telle, c'est celui qui fait la piqûre. Il l'a piquée, et il l'a tuée. Il tue très vite"

"Tu vas voir des sortes de plaies, blanchâtres, comme des écorchures ça fait mal, c'est blanc. Il y a aussi des petits boutons"

"J'ai une belle-sœur qui a eu cette maladie. On était assise comme ça et elle a giflé son bébé et l'a jeté par terre. Très vite, on s'est levé et on l'a attachée. Elle avait les yeux écarquillés" 


\section{lors des consultati
le dispensaire :}

"Si je constate que c'est une femme qui accouche pour la première fois, je l'amène au dispensaire, on nous a dit d'amener ce genre de cas comme ça accoucher au dispensaire"

51 Quant au fait que l'accoucheur soit un homme et non une femme, cela a été parfois signalé comme source de gêne (de "honte") pouvant empêcher certaines femmes d'accoucher en dispensaire.

"Accoucher devant l'infirmier constitue une honte parce qu'il n'est pas mon mari"

"Moi, je ne peux même pas le regarder si je le rencontre" 
"A l'heure actuelle, on amène beaucoup de gens et ce sont les infirmiers (hommes) qui mettent la main au niveau de la femme pour renverser le bébé. C'est pour cette raison, d'ailleurs, que les femmes n'aiment pas aller accoucher au dispensaire. Mais si c'est une femme comme elles, tu vois bien qu'il n'y aura pas de problème, elle pourra les examiner. Par contre si ce sont les hommes, elles ont du mal à accepter ce toucher qu'ils font. C'est pourquoi des femmes refusent d'aller au dispensaire pour accoucher"

"L'accouchement à domicile est plus honorable que celui effectué au dispensaire, à domicile cela se passe entre femmes, tandis qu'au dispensaire c'est un homme qui fait l'accouchement"

De l'avis d'un marabout ceci est même inconcevable :

"Ce sont les Blancs qui ont amené des choses comme ça. Le Coran a séparé l'homme et la femme, nul ne doit aller vers l'autre sauf si c'est une nécessité. La femme qui est malade doit être soignée par une femme. De même l'homme qui est malade doit être soigné par un homme. Le Coran n'a pas autorisé le contraire. Depuis l'époque $\mathrm{du}$ Prophète, cette interdiction existe. Celui qui l'outrepasse a contrarié le Prophète et celui qui a contrarié le Messager a contrarié Dieu, c'est pourquoi nous traitons la personne de cafre".

Ceci étant, ce type de discours n'a débouché nulle part, sur nos lieux d'enquête, sur un boycott systématique de l'accouchement en dispensaire. Il est vrai qu'il n'y avait pas dans ces villages de groupes intégristes (là où ils sont implantés, les "isalistes"-membres de la confrérie Isala - interdisent à leurs femmes d'être accouchées ou examinées par des hommes) ${ }^{16}$.

Parfois aussi c'est un argument financier qui est avancé pour éviter l'accouchement au dispensaire.

"On dit que les femmes allant accoucher au dispensaire doivent payer 500 FCFA.

Pour cette raison, les femmes ne vont pas accoucher au dispensaire"

Cependant les gens sont conscients que diverses complications de l'accouchement peuvent trouver une solution au dispensaire.

"Il y a moins de problèmes au dispensaire. Même si l'enfant est mort dans le ventre, ils peuvent le savoir, alors que nous nous ne pouvons pas le savoir"

"Moi, dès que je sens une difficulté, je me dirige vers le dispensaire pour avoir du secours"

Enfin, il faut noter que l'accouchement au dispensaire entraîne d'autres types de soins au nouveau-né que l'accouchement à la maison. L'infirmier(ère) s'intéressera d'avantage, et d'abord, à l'enfant : examen, vaccination, pesée. Il le portera directement au sein de la mère après avoir ordonné de nettoyer le sein avec de savon et de l'eau. Le placenta sera donné à la matrone ou aux vieilles femmes pour son enfouissement traditionnel. Sauf complications, la mère quitte en général le dispensaire avec son bébé dans les heures qui suivent l'accouchement pour rentrer chez elle et être prise en charge selon les habitudes (les 7 jours en chambre précédant le baptême, et les "40 jours").

Les matrones

57 Alors qu'il existait partout en milieu rural des guérisseurs relativement spécialisés avant la colonisation (pour les fractures, les morsures de serpent, la lèpre, etc.), on ne trouvait pas de fonction d'accoucheuse véritablement spécialisée à l'échelle du village. L'accouchement était une affaire de famille ou de voisinage (prenant même souvent une forme solitaire, les femmes se vantant alors d'avoir accouché seules). Certaines vieilles femmes plus habiles ou plus expérimentées prêtaient main forte en cas de 
besoin. C'était essentiellement autour de la "coupure du cordon" que telles ou telles se spécialisaient partiellement.

En pays hausa, dans la région enquêtée, le terme aujourd'hui consacré est angozoma (ou ingozama), d'origine indéterminée. Il s'applique, à l'heure actuelle, à la fonction de "matrone" proprement dite, au sens officiel du terme. L'avènement de cette fonction, et sa vulgarisation par les services de santé, semble avoir un lien avec l'utilisation du terme angozoma.

"Il y avait des angozammai, c'est-à-dire une vieille femme en laquelle tu as confiance et qui est sérieuse. Si ta femme accouche, tu la fais venir (ou) elle vient, elle arrange, elle coupe le cordon et tout. Durant toute cette semaine, elle vient voir le bébé, s'enquérir de sa santé. On pose la marmite, on lave, on fait tout. Si le soir vient, on amène à cette vieille femme le repas qu'on a préparé. C'est tout. Après cette semaine, elle revient. Elle vient, elle s'assoit, elle prend le bébé dans ses bras et on lui fait la fatiya. Alors, on lui donne son dû. On amène une peau, on lui donne, on amène 15 CFA si c'est un garçon $(3 \times 5)$, et si c'est une fille 20 CFA $(4 \times 5)$. (...) On prend un peu de mil dans une petite calebasse, on lui verse. On amène du piment et $\mathrm{du}$ sel, du natron. Tout cela, on amène dans une petite calebasse et on lui donne. Ainsi, on récompense la vieille. C'est ce que nous avons vu les parents faire" .

Il n'existe pas de mot proprement zarma pour "matrone". Aujourd'hui on utilise donc systématiquement le terme d'origine française matron. Autrefois, on parlait plutôt de "coupeuse de cordon" (fuuma-dumbu-ko). Pour certaines d'entre elles, leur mère le faisait déjà. Mais en ce cas il ne faut pas y voir l'effet d'une quelconque norme de transmission "par héritage" ou de lignée. Opportunités d'apprentissage familial et dispositions personnelles entrent ici en jeu. Il n'y a ni véritable spécialisation professionnelle ni savoir ésotérique.

"Auparavant, couper le cordon (fuuma dumbuyon), cela s'héritait-il de mère en fille? Il n'y avait là rien de cela, c'était une simple question de s'y mettre, celle qui avait commencé à couper des cordons, c'était elle qu'on appelait, elle coupait le cordon, elle lavait l'enfant, elle lavait la mère, elle enterrait le placenta (coro) (KABA)"

"Avant qu'on n'ait écrit mon nom comme matrone, je coupais le cordon, je lavais la mère et l'enfant, ce n'était pas que je l'avais hérité de ma mère, faire cela c'est une question de n'être pas émotif (littéralement : d'avoir "la rate mûre")

"A la fois je l'ai hérité de ma mère et je ne l'ai pas hérité. Ma mère coupait déjà les cordons. Dieu a fait que moi aussi j'ai eu le cœur assez bien accroché pour le faire (littéralement : avoir "le cœur mûr", bine niney). Avoir le cœur bien accroché, cela veut dire que, même si tu as pitié de la personne, une fois que tu as décidé de faire ce travail, que ça te plaise ou non tu vas couper le cordon"

Nous appellerons "accoucheuses traditionnelles" ces femmes plus compétentes ou motivées que d'autres, qui continuent souvent en milieu rural à assister les parturientes, et nous les distinguerons donc des "matrones" proprement dites, issues de leurs rangs mais ayant eu une "formation"- cette dénomination de matrone a en effet pour les populations pris un sens quasi officiel -, bien que les unes et les autres fassent en fait maintenant à peu près les mêmes gestes.

"Il n'y a pas de changement aujourd'hui, c'est comme avant, sauf que l'on écrit

désormais la naissance sur un papier" .

61 Ce sont les services de développement qui ont introduit la fonction de "matrone" (les premières ayant été "formées" par l' "animation rurale" peu après l'indépendance, à partir de 1965, le relais ayant été ensuite pris par les structures de santé et les ONG ${ }^{17}$ ). En ce sens, les matrones sont donc le produit d'une intervention extérieure et apparaissent comme des "agents de santé communautaire", investis par les services 
officiels, au même titre que les secouristes. Matrones et secouristes sont même parfois appelés lokotoro, "docteur", ou mazor (major, c'est-à-dire infirmier-chef). On devient "matron" quand on a participé à une "formation" à l'extérieur et que, à cet effet, on a eu son nom porté sur un registre. "Matron" fait partie de ces quelques fonctions semi-officielles villageoises, imposées ou impulsées par l'extérieur (Etat ou intervenants) qui se sont succédées ou additionnées depuis l'indépendance aux côtés des chefs de village: komita (responsable du comité du parti unique), animasio ("animateur" désigné du village), samaria (responsable de l'association officielle de la jeunesse), sekurist (secouriste), etc.

62 Lorsqu'il s'est agi de recruter ces matrones à l'échelle des villages, et de les envoyer éventuellement en "stage" (15 jours, aux débuts ${ }^{18}$, parfois moins aujourd'hui), les volontaires ne se sont pas bousculées: la méfiance, et aussi le sentiment qu'il allait s'agir d'une fonction coûteuse en temps et sans rémunération intéressante, ont découragé les candidatures. Aussi la forte proportion de matrones ayant des liens de parenté avec le chef de village (15/20 dans notre enquête) ne manifeste-t-elle pas en l'occurrence un quelconque népotisme villageois, mais au contraire le fait que, sommés de désigner quelqu'un, nombre de chefs ont demandé à leur femme, leur nièce, leur tante ou leur sœur de bien vouloir se dévouer...

"Des docteurs (lokotoro) sont venus, ils ont dit qu'ils voulaient des femmes du village pour apprendre le travail des docteurs (lokotoro tarey) (...) Un jour ils sont arrivés pour nous prendre pour nous amener (en stage) à Dosso. Je n'en avais pas du tout envie. Mais c'était le chef de village qui m'avait demandé d'y aller, et je ne l'ai pas contrarié parce que c'est mon oncle"

Parfois, en particulier dans les villages où il y avait une "coupeuse de cordon" plus ou moins attitrée et de bonne réputation, il n'y pas eu débat, et celle-ci a été en quelque sorte désignée d'office par les femmes ou par la population.

"Je ne me suis pas proposée. C'est le village qui m'a proposé"

$\mathrm{Au}$ total, désignées par consensus ou par le chef, beaucoup de matrones sont d'anciennes "accoucheuses traditionnelles".

"Avant de devenir "docteur"(lokotoro),je coupais déjà les cordons"

"Avant même la création du dispensaire, je faisais ce travail, avant notre formation"

Mais, désormais, elles doivent leur titre de "matron" à leur départ en stage, quelle que soit la durée ou l'ancienneté de celui-ci.

"La formation que nous avons reçue a été dirigée par une Blanche. Elle nous a montré la tête et nous a demandé ce qu'elle comportait. Nous avons répondu qu'il y a les yeux, les oreilles, le nez, les dents. Elle a demandé pour les mains. Nous avons dit qu'il y a 5 doigts. Et pour les pieds, nous avons dit qu'il y a 5 doigts. C'est comme ça qu'elle nous a formées"

66 Nous n'avons pas compétence pour juger de la qualité des formations reçues. Elles semblent variables, de 3 jours minimum à 15 jours maximum, parfois effectuées il y a plus de 20 ans. Rares sont les cas de recyclages.

67 En tout cas, notre enquête, menée auprès de 20 matrones et de 26 femmes ayant récemment accouché dans les 8 villages investigués, enquête dont les conclusions recoupent les diverses observations que nous avons faites depuis plusieurs années dans 
bien d'autres villages nigériens, fait ressortir clairement que le rôle des matrones se limite pour l'essentiel à quatre fonctions possibles :

- une assistance à l'accouchement, qui, le plus souvent, n'offre guère de compétence supplémentaire particulière par rapport aux accoucheuses "traditionnelles" (dans la mesure où les gestes innovants introduits avec succès par le biais des matrones sont désormais reproduits aussi par les accoucheuses traditionnelles : cf. infra);

- un accompagnement éventuel de la parturiente vers le dispensaire en cas de problème, avec souvent une participation, parfois déterminante, à la décision de ce type d'évacuation de proximité

- une déclaration de naissance au dispensaire pour les enfants nés à la maison ;

- un rôle, pour certaines, d'auxiliaires d'un infirmier ou d'une sage-femme, permettant l'acquisition de compétences nouvelles, parfois investies aussi dans des accouchements "à la maison".

On peut présenter ainsi la diversité des situations que l'on rencontre, à partir de l'investigation systématique que nous avons menée sur 55 cas d'accouchements récents.

En cas d'accouchement "à la maison "(généralisé dans les villages sans dispensaire -la majorité -, et encore fréquent, sauf usage de la contrainte, dans les villages avec dispensaire)

Il y a alors diverses possibilités :

71 Si l'accouchement se passe normalement en particulier si le travail n'est pas considéré comme trop long

- Soit on n'a pas recours à la matrone (cas de Mashe Jan Baushi et Azazala, où chaque famille a ses accoucheuses traditionnelles)

- Soit on l'appelle seulement après l'expulsion, essentiellement comme "coupeuse de cordon" : c'est le cas de plus de la moitié des accouchements auxquels les matrones interrogées ont procédé ces derniers mois (32/55). En ce cas elle lavera aussi le placenta et l'enterrera, elle lavera le nouveau-né et la mère, mais ce sont des tâches que les autres vieilles femmes peuvent faire aussi.

"C'est après l'accouchement qu'on nous appelle, pour couper le cordon"

"Le plus souvent, tu trouves le bébé déjà à terre, parfois le placenta aussi. Tu coupes le cordon et tu t'occupes du placenta"

Soit elle est appelée pendant le travail, parce qu'elle est voisine ou que c'est une parente, parce qu'elle a particulièrement bonne réputation, ou parce que l'habitude commence à se prendre chez les plus jeunes de demander son assistance pour l'accouchement en raison des conseils des infirmiers lors des consultations pré-natales

"Certaines nous appellent une fois qu'elles ont accouché, certaines nous appellent pour l'accouchement"

Dans les trois cas, c'est la matrone qui devra aller faire ultérieurement la déclaration de naissance au dispensaire le plus proche (éventuellement avec la mère, s'il y a un problème; nécessairement avec le nouveau-né, qui sera examiné et vacciné par l'infirmier). Cette fonction de type bureaucratique est sans doute la principale raison $\mathrm{du}$ recours aux matrones, et est à l'origine du fait que, dans certains villages, elles aient désormais acquis le "monopole" du "coupage de cordon".

"Si une femme accouche, c'est pour pouvoir obtenir le certificat de naissance qu'elle nous appelle" 
"Si ce n'est pas nous qui allons déclarer la naissance, l'enfant ne pourra avoir d'acte de naissance. Quand une femme a accouché, c'est nous qui amenons son carnet de consultations pré-natales (neesi tira) au dispensaire pour l'écriture".

"Les femmes ont peur car si elles vont au dispensaire l'infirmier va leur demander où est la matrone". de Serkin Hausa) et servent en fait d'auxiliaires (avec "tours de garde") aux infirmiers(ères) qui procèdent à l'accouchement. Seules ces matrones, parce qu'elles sont confrontées à de fréquents accouchements (à la différence d'une matrone ou d'une "accoucheuse traditionnelle" de village, qui n'assistent que peu de femmes chaque année), et qu'elles observent les pratiques des infirmiers(ères) pendant tout le travail et en cas de problèmes, peuvent éventuellement acquérir peu à peu la compréhension de certaines complications, voire la maîtrise de certaines manœuvres obstétricales (percement de la poche des eaux, version par manœuvre externe, mesure de la dilatation, réanimation du nouveau-né). Certaines peuvent réinvestir ces compétences dans des accouchements "à la maison" (cf. matrone de Belendé).

"Tout ce que je sais je l'ai appris au dispensaire. En fait, les matrones qui ont fréquenté le dispensaire en savent beaucoup plus que celles qui sont en brousse (...) Avant, si un enfant ne respire pas à la naissance, on disait qu'il était mort et on allait l'enterrer. Moi, maintenant, je lui souffle dans la bouche et dans les narines, je lui masse la poitrine, je lui plie les pieds sur le ventre. Si la respiration peut venir, elle viendra. J'ai vu faire l'infirmier, et je fais comme lui"

Les tâches spécifiques des matrones particulière en obstétrique (aucune n'a appris à mesurer la dilatation du col, par exemple). En cas de complication, elles n'ont à leur disposition (sauf celles qui se sont formées "sur le tas" au dispensaire) que - outre les prières - quelques truc traditionnels (on a vu ci-dessus lesquels), et ne jouent donc de rôle particulier que pour éventuellement recommander l'évacuation vers le dispensaire rural.

Par contre, elles ont appris quelques gestes usuels simples, non pratiqués traditionnellement, et qui demandent des objets ou des produits spéciaux : couper le cordon avec une lame de rasoir, ne pas laisser le nouveau-né par terre, désinfecter la plaie, mettre de l'argyrol (si on en a) dans les yeux du nouveau-né, dégager les voies respiratoires, amener le nouveau-né au dispensaire pour les vaccinations. Couper le cordon avec une lame et ne pas laisser l'enfant naître à même le sol sont en fait les deux grandes innovations partout passées dans les mœurs. 
"Avant, il n'y avait pas de lame de rasoir, on coupait le cordon avec une tige de mil"

"Avant, la femme s'agenouillait par terre et accouchait, l'enfant tombait sur le sol. Maintenant nous ne le laissons pas toucher terre, nous l'interceptons avant" .

\section{deux cas, elles sont fréquemment usagées.}

"La lame que nous avons achetée, après avoir coupé le cordon, nous la lavons et la rangeons jusqu'à une prochaine occasion".

"Nous laissons la lame chez la femme. C'est à elle. Chacune achète sa lame"

81 Aujourd'hui, même là où il n'existe aucune matrone (là où il n'y a que des accoucheuses traditionnelles), le cordon se coupe désormais avec une lame, et l'on accouche agenouillée sur une natte. A cet égard le "message est passé". Mais, de ce fait, en dehors de leur "officialisation", rien ne distingue non plus les matrones des autres accoucheuses non "formées", d'autant que les autres éléments appris au cours de leurs formations restent en général lettre morte pour diverses raisons ${ }^{19}$. Par exemple, l'accouchement en position gynécologique, qui a souvent été recommandé au cours des stages, n'est évidemment jamais pratiqué à la maison. La position agenouillée, préférée par les femmes, est seule utilisée.

En ce qui concerne le désinfectant et l'argyrol, si 6 matrones sur 20 interrogées avaient l'un ou l'autre produit, seules 3 matrones sur 20 détenaient les 2 produits (sur ces 3, 2 venaient de les obtenir à la suite d'un stage, et ne s'en servaient pas, le village ne faisant jamais appel à elles...). Manifestement le ré-approvisonnement ne se fait pas facilement auprès des dispensaires. La raison financière est le plus souvent invoquée

- "Tu as la caisse à pharmacie qu'on t'a donnée?

- Elle est là

- Tu t'en sers?

- Je m'en sers, sauf que maintenant il n'y a plus de produits

- Tu n'en achètes pas?

- Avec quel argent ?"

Une matrone n'en vaut pas une autre

Le facteur personnel intervient de façon non négligeable. Non seulement la compétence varie largement, mais aussi interviennent des facteurs d'ordre personnel, social, moral.

On ne fera pas appel à certaines matrones parce qu'elles sont considérées comme médisantes:

"La matrone de notre quartier, celle qui est devenue aveugle, les gens en ont peur, car elle parle trop, on lui a dit d'être plus discrète, elle ne l'a pas fait, aussi même dans notre famille personne ne va plus l'appeler pour un accouchement, bien que ce soit une cousine"

"On n'aime pas celle qui est médisante, il faut quelqu'un de sérieux, qui tait tout ce qu'elle voit"

D'autres, on dira que leur "main n'est pas bonne" :

"Pour être matrone, c'est une question de "main", pour certaines, le travail est plus facile que pour d'autres, c'est un don"

Telle matrone refusera d'accoucher les filles non mariées

"Si on m'appelle pour faire accoucher une fille qui est tombée enceinte n'importe comment, cela m'embarrasse beaucoup, j'ai même refusé d'y aller, car je sais que si les marabouts l'apprennent, ils diront que ce n'est pas le chemin qu'il faut suivre et qui est dans le Coran (...) Avant, même pour le premier vagissement d'un bâtard, les gens se sauvent, car on dit que celui qui l'entend ira en enfer (...). C'est l'infirmier-major qui m'a obligé à m'en occuper, il dit que nous n'avons pas le droit 
de les discriminer ainsi. Pourtant, y aller, c'est un péché, mais c'est sur l'infirmier-major que le péché sera, puisqu'il m'a obligé" sont en fait fort variables. D'une façon générale, elles vont de 100 FCFA à 1000 FCFA, sans compter le don éventuel de mil, de savon, et de viande le jour du baptême. Dans certaines sessions de formations de matrones, les instructrices ont même affirmé qu'une partie du mouton du baptême leur revenait de droit. Ceci est bien loin d'être appliqué, et est même combattu par certains marabouts. Au total, si la tendance générale des matrones est de se plaindre, ou d'évoquer un temps révolu plus généreux, les propos sont en fait assez divers :

"Avant on nous donnait deux pagnes, et mille francs si c'était le premier accouchement de la femme, on nous donnait une calebasse de mil, de la cola, du parfum ; aujourd'hui les gens disent qu'ils n'ont rien, on nous donne 500 FCFA"

"Certains nous donnent quelque chose, certains nous disent d'attendre qu'ils repartent à la maison, et puis plus rien ; il faudrait qu'ils soient obligés de donner, que le chef impose qu'on nous paye pour notre travail"

"On ne me donne rien, je coupe simplement le cordon, parce que ce sont mes voisines ou mes parentes"

"Avant, on m'apportait les côtelettes de l'animal du baptême ; on disait que c'était la coutume, parce que je massais les côtes de la femme en couches; un nouveau marabout est venu, il a supprimé ma part et celle du chef de village, il a dit : "Est-ce que c'est toi qui as acheté le mouton ou la chèvre? pourquoi tu veux qu'on te donne les côtelettes ?". Je ne vais plus au baptême, pour que les gens ne pensent pas que j'y vais pour chercher quelque chose"

De façon générale, les matrones soulignent la contradiction entre leur fonction quasi-"officielle" (choisies "par le gouvernement", "formées" par lui, assumant à sa demande une tâche d'intérêt collectif), et leur absence de salaire. Elles revendiquent un statut clair, avec ses avantages symboliques et matériels.

Les évacuations

L'évacuation en cas de complication est évidemment le principal moyen de réduire la mortalité et la morbidité maternelle grave dans les campagnes. En fait l'évacuation comprend en général deux étapes.

Nous parlerons d'évacuation A dans le cas d'une évacuation où un travail a commencé "à la maison", et où des difficultés obligent à amener la parturiente au dispensaire (que celui-ci soit situé dans son village ou dans un autre village). Nous parlerons d'évacuation B (référence) pour les évacuations partant d'un dispensaire vers une 
structure de référence (on aura parfois deux références successives). Il y a évidemment une différence fondamentale entre ces deux niveaux. L'évacuation A est décidée par des acteurs villageois, dans le cas d'un accouchement commencé localement, et consiste à amener la parturiente dans un dispensaire rural (en général en charrette). L'évacuation $B$ est une évacuation médicale, décidée par un infirmier(ère) ou une sage-femme, vers une structure hospitalière urbaine (en général en voiture). Bien sûr une évacuation $\mathrm{A}$ peut déboucher sur une évacuation $B$.

Nous avons présenté en préambule un cas d'évacuation. En voici deux autres, d'ordre différent, parmi les 25 recensés

La mort de Mariama

Mariama, agée de 37 ans, est mère de 6 enfants, et habite au quartier Belande Kayna, du village de Belende, où il y a un dispensaire. Elle n'a jamais rencontré de problèmes avec ses grossesses antérieures. Elle les conduisait jusqu'à terme et a toujours accouché dans la discrétion, autrement dit c'était après l'accouchement qu'elle avertissait la matrone pour que celle-ci vienne couper le cordon. Sa belle-mère affirme que "Mariama n'a jamais manqué les consultations pré-natales". Son mari est parti en exode lorsqu'elle en était au $5^{\text {ème }}$ mois de sa nouvelle grossesse. Le jour du drame, sa belle-mère raconte : "Mariama, dans l'après midi, a pilé sa farine pour le repas du soir, après elle s'est retirée dans sa chambre. Au crépuscule, comme j'ai remarqué son absence de la cour, j'ai envoyé sa fille s'enquérir de ses nouvelles. C'est ainsi qu'elle est revenue me dire que sa mère souffrait de maux de tête. L'instant d'après, Mariama m'a fait appeler en disant qu'elle est en train de perdre du sang et qu'elle n'a jamais vu pareil cas. J'ai donc aussitôt envoyé sa fille avertir la sœur de Mariama qui est au quartier Zarmay."

Cette dernière arrive, constate la situation, et appelle la matrone, qui habite à proximité. Celle-ci vient aussitôt, et, impressionnée par l'hémorragie, demande qu'on apprête une charrette pour évacuer la parturiente, sans intervenir.

Mariama ne peut monter d'elle-même dans la charrette tant elle est épuisée par l'hémorragie. Parmi les accompagnants, il y a deux de ses sœurs, un frère contacté en cours de chemin, et la matrone. La présence du frère est justifiée ainsi par la sœur de Mariama : "en cas de besoin, on peut l'envoyer chercher telle ou telle chose en ville, ce qu'une femme ne peut faire vue l'avancée de la nuit".

Après l'admission de Mariama au dispensaire, sa sœur va demander du hantum hari (eau ayant lavé des écritures coraniques) auprès d'un de leurs frères, marabout : "J'étais à la mosquée pour la prière de $20 \mathrm{~h}$ lorsque notre sœur est venue m'informer et m'a demandé de les aider avec le "Irkoy Nwaray"; pour des cas pareils, il y a des verset spéciaux". Mais l'eau sainte, pas plus que le sérum de l'infirmière, n'a d'efficacité. Le sang continue de couler. L'infirmière fait sortir l'enfant, qui est mort-né, la matrone coupe le cordon, et le cadavre est enterré immédiatement.

L'infirmière ne désarme pas, et fait des va-et-vient entre sa maison et le lit de Mariama au dispensaire, pour surveiller la perfusion. La première étant finie, elle en place une seconde. Aucune tentative d'évacuation vers l'hôpital ne semble avoir été envisagée. Mariama dit à sa sœur de lui enlever la perfusion, car elle sait que la mort arrive. Aux environs de 23h, elle rend l'âme.

L'enfant mort-né de Santou

101 Malgré sa grossesse avancée, Santou, qui habite Belende, et dont c'est la première grossesse, vaque à ses occupations quotidiennes. "Si tu vas à la consultation, l'infirmier 
te dit de ne pas faire les travaux pénibles tels que prendre un seau d'eau, alors qu'au village, tant que tu te portes en bonne santé, tu vas travailler. Si tu refuses de le faire on te traitera de paresseuse, tu seras couverte de honte, car autour de toi, toutes tes camarades qui sont dans le même état travaillent" .

Un jour, Santou a de fortes douleurs au ventre, mais cela ne l'empêche pas de continuer ses tâches domestiques. Santou ne pense pas qu'il s'agisse du début du travail : "j'ai cru que c'était dû aux travaux que je venais d'effectuer au champ".

Les douleurs redoublent d'intensité et s'accompagnent d'une perte de sang. Cela dure toute une demi-journée.

Elle se confie à une amie qui, elle, a déjà accouché, et qui lui dit d'aller au dispensaire. Santou s'y rend dans l'après midi en compagnie de son amie. Là l'infirmier fait un toucher et lui donne quelques comprimés. Santou repart ensuite et va chez sa mère, dans un quartier voisin, Sillance. La situation les inquiète toutes les deux. Toute la nuit Santou a mal au ventre. Son mari est mis au courant, mais ne s'alarme pas, parce que c'est le travail. Le lendemain Santou et sa mère vont au dispensaire. L'infirmier l'examine à nouveau, il lui fait une injection, et lui prescrit une ordonnance. A l'époque Belende ne disposait pas de dépôt pharmaceutique, le dépôt se trouvait à Falmey, à 17 $\mathrm{km}$. Le mari part à Falmey y chercher les médicaments. Entre temps Santou continue de souffrir. Le major lui fait encore une injection pour accélérer la délivrance, mais en vain. "Il m'a fait la piqûre à midi, je me suis alors couchée, vers l'après-midi l'enfant a tenté de sortir, je me suis agenouillée, l'enfant s'est retourné, sa main est sortie, l'infirmier a tout fait mais il n'a rien pu faire, parce que l'enfant est sans vie".

Devant l'échec de ses tentatives, l'infirmier décide alors d'évacuer la parturiente sur Birni : "l'enfant s'est placé de travers (a gant). Il se charge de négocier gratuitement un véhicule de transport dans lequel Santou, sa mère et l'infirmier prennent place (normalement le transport de Belende à Birni coûte environ 750 FCFA).

Ils arrivent à Bimi avant la tombée du soir. Là, il n'y a que des infirmiers, pas mieux formés ou équipés que l'infirmier de Belende. "Presque tous les infirmiers ont essayé, l'enfant n'a pu se retourner, sa main qui était sortie n'a pu être refoulée, la peau s'est même arrachée; aux environs de la prière du soir, on m'a évacuée sur Dosso. "L'infirmier de Belende est retourné à son poste, laissant Santou et sa mère faire le reste de l'itinéraire $(39 \mathrm{~km})$.

107 A Dosso, Santou est immédiatement introduite en salle d'opération pour y subir une césarienne. "Lorsque les gens se préparaient à me faire l'opération l'enfant s'est retourné, Dieu a ainsi facilité les choses, l'enfant est tombé, mais il était mort".

, qui était absent pendant l'évacuation sur Birni, s'est entretemps rendu à Birni, puis a continué pour la rejoindre à Dosso tard dans la nuit. Le lendemain il va enterrer l'enfant au cimetière. Santou est hospitalisée pendant 6 jours durant lesquels on lui a fait des injections et donné des comprimés.

Le mari dit avoir dépensé 15000 CFA. Durant tout l'épisode la mère de Santou a fait dire des versets coraniques.

Le parcours-type

110 En cas de difficultés, si l'accouchement se passe à la maison, la matrone est normalement appelée, et joue un rôle dans la décision d'évacuation, dans le concert familial. Le plus souvent la matrone n'essaie aucune intervention, et dirige la femme directement vers le dispensaire, le parcours se faisant généralement en charrette 
(évacuation A). L'évacuation doit avoir l'aval du mari. Plusieurs membres de la famille accompagnent la femme en travail. Au dispensaire, l'infirmier(ère) procède le plus souvent à l'accouchement. Cependant, s'il ne réussit pas, il décidera de référer la parturiente à la Circonscription médicale (CM) dont dépend le dispensaire (évacuation B). Les frais de transport sont à la charge de la famille. Parfois l'infirmier du village fait appel à un véhicule de transport en location, parfois il va chercher l'ambulance dans un poste médical plus ou moins éloigné. Les frais exigés peuvent s'élever jusqu'à 15.000 FCFA, ce qui est beaucoup pour des paysans.

"C'est l'infirmier qui va chercher (en mobylette) l'ambulance jusqu'à F (à plus de 15 $\mathrm{km}$ ). Il faut que tu payes les frais d'essence (de la mobylette, puis de l'ambulance). Si tu n'as pas d'argent, tu vas mourir".

La mobilisation de moyens financiers à travers le réseau familial dans le village est souvent la première phase de l'évacuation. C'est un des rôles que les hommes jouent dans les évacuations (A et B). Il faut qu'il y en ait au moins un qui soit accompagnant. En effet :

- Ils doivent débourser l'argent du transport car "un voyage comporte plusieurs surprises, c'est pourquoi il faut toujours se munir d'argent pour partir".

- "Pour certaines commissions, les hommes sont plus efficaces que les femmes. Les femmes, elles, sont efficaces dans l'assistance. Si par exemple, il s'agit d'aller chercher quelque chose tard dans la nuit, c'est un homme qui est indiqué (...) Il se peut aussi qu'il y ait une mort, c'est 1 'homme qui fera le nécessaire pour l'enterrement" .

112 Par contre, il semble que le chef de village soit peu ou pas concerné. En fait, l'unité d'interconnaissance pertinente est le quartier. Les gens d'un même quartier savent ce qui se passe dans leur quartier (y compris les accouchements, et leurs éventuels problèmes), et beaucoup moins ce qui se passe dans les autres quartiers du même village.

113 Un des problèmes des évacuations B est lié au passage obligatoire par la structure supérieure, selon le modèle "dispensaire rural (CSI) circonscription médicale hôpital régional". Or seul l'hôpital est équipé pour des césariennes, et souvent l'échelon intermédiaire n'a guère plus de moyens et de compétences à sa disposition que le dispensaire rural : cependant il faut passer par lui (avec des parcours de plusieurs dizaines de kilomètres, et les conséquences que cela comporte en termes de coûts et de délais) avant d'être dirigé sur l'hôpital.

114 On verra maintenant que l'analyse des 25 cas de complications avec évacuations que nous avons recueillis révèle quelques écarts par rapport à ce parcours type, tout en le confirmant dans les grandes lignes.

Analyse des 25 cas d'évacuations

Motifs ( $\mathrm{n}=25)$

Les 25 évacuations ont eu les causes suivantes :

- 13 = "travail long" (dont 1 correspond à une mauvaise présentation de la tête non perçue par l'entourage). C'est donc de loin la principale cause d'évacuation par les familles. Il semble cependant difficile de fixer une durée standard à partir de laquelle le travail est perçu comme "trop long" et inquiétant ${ }^{20}$.

- 4 = mauvaises présentations ( 2 de la tête, 1 par la main, 1 par le pied)

- 3 = hémorragies (dont 1 cas avec décès de la mère et de l'enfant)

- 3 = rétention placentaire (dont 2 après avortement, et 1 cas non perçu comme rétention) 
Evacuations B ( $\mathrm{n}=5)$

Conclusion d'efficacité.

Enfin, nous avons eu à connaître d'1 cas d'éclampsie (diagnostiqué par l'infirmier, et non connu par l'entourage)

Décision d'évacuation $(\mathrm{n}=22)$

- 8 fois elle a été prise à l'initiative de la parturiente elle-même (ce qui montre que souvent celle-ci ne joue pas un rôle passif et que son inquiétude est prise en compte)

- 6 fois à l'initiative de la matrone

- 4 fois à l'initiative du mari (1 fois de son père, le mari étant absent)

- 4 fois à l'initiative de la mère, de la belle-mère ou de la grand-mère

- Moyens de transport pour les évacuation A $(n=25)$

- 13 fois elle s'est faite en charrette ( dont 7 avec la charrette familiale, 4 avec une charrette empruntée et 2 avec une charrette louée)

- 5 fois elle s'est faite en voiture (= village au bord d'une route ; la place en voiture a été 1 fois gratuite, 4 fois payante)

- 7 fois elle s'est faite à pied (= dispensaire dans le village)

D'autre part, en 7 occasions, la matrone a fait partie des accompagnants

Sur les 5 évacuations B, deux ont transité par les Circonscriptions médicales (CM), pour être ensuite ré-orientées vers l'hôpital départemental (CHD). En 3 cas la matrone a suivi. En 2 occasions il a été fait appel à une ambulance.

Si l'accouchement se déroule normalement, le fait qu'il se produise à la maison dans des conditions traditionnelles semble parfaitement acceptable, mais par contre, en cas de difficultés sérieuses, le seul recours est l'évacuation, dans la mesure où les "savoirs populaires en matière d'obstétrique sont, on l'a vu, fort limités d'un point de vue bio-médical, et ne permettent pas de faire face à des complications de façon efficace. Or, partout l'évacuation est un problème, qui devient dramatique pour les villages isolés et éloignés des routes et dispensaires.

D'autre part, des décennies de formation de "matrones" par des projets ont produit peu d'effets, hormis la généralisation de l'usage de lames de rasoir pour couper le cordon, et n'ont que très peu contribué à lutter contre la mortalité maternelle: seule leur éventuelle participation aux décisions d'évacuation peut sans doute être créditée

Mais les évacuations vers des dispensaires ruraux ne règlent qu'une toute petite partie des facteurs de mortalité maternelle, d'autant plus que les personnels de santé y sont insuffisamment formés, et dépourvus de matériel obstétrical minimum. Une formation spécialisée de ces personnels semble constituer l'un des éléments prioritaires d'une solution. L'autre consiste évidemment dans l'amélioration des moyens d'évacuation rapide et directe vers des plateaux techniques équipés pour des césariennes.

Sur ces diverses questions, l'étude socio-anthropologique n'apporte sans doute pas de révélations, mais elle renforce cependant, ou confirme, les hypothèses et les propositions de divers spécialistes de santé publique ou d'obstétrique. Tout le problème est le passage à l'acte en matière de politique de santé nationale ou de coopération internationale, qui est loin d'être fait. 


\section{BIBLIOGRAPHIE}

Attama, S., Seroussi, M., Kourguéni, AI., Koché, H. \& Barrère, B. 1999. "Enquête démographique et de santé, Niger 1998". In : Care International Niger/Demographic and Health Surveys, Niamey.

Jaffré, Y. \& Moumouni, A. 1993. "Etre aveugle". La cécité, entre une définition épidémiologique et sociale". Bulletin de la Société de Pathologie Exotique 86(4) : 295-299.

Jaffré, Y. \& Moumouni, A. 1994. "L'importance des données socio-culturelles pour l'accès aux soins et l'observance des traitements dans la lèpre. L'exemple du pays zarma au Niger". Bulletin de la Société de Pathologie Exotique 87 : 283-288.

Jaffré, Y. \& Olivier de Sardan J.P. 1996. "Tijiri : la naissance sociale d'une maladie". Cahiers des Sciences Humaines 31(4) : 773-795.

Jaffré, Y. \& Olivier de Sardan, J.P. 1999. La construction sociale des maladies. Les entités nosologiques populaires en Afrique de l'Ouest. Paris : PUF.

Jaffré, Y. \& Prual, A. 1993."Le corps des sages femmes, entre identités professionnelle et sociale". Sciences Sociales et Santé 11 (2).

Mangay-Maglacas, A \& Pizurki, H. 1983. Les accoucheuses traditionnelles dans sept pays. : Genève : OMS.

Olivier de Sardan, J.P. 1982. Concepts et conceptions songhay-zarma (histoire, culture, société. Paris : Nubia.

Olivier de Sardan, J.P. 1994."La logique de la nomination. Les représentations fluides et prosaïques de deux maladies au Niger". Sciences Sociales et Santé 12(3) : 15-45.

Souley, A. 1999."Trois entités hausa parentes : zahi, sanyi et shawara". In Jaffré \& Olivier de Sardan (eds).

Vangeenderhuysen, C. \& Mounkaïla, N. 1997. Enquête multicentrique MOMA sur la mortalité et la morbidité grave au cours de la grossesse. Résultats du Niger. Niamey : Ministère de la Santé Publique

Verderese, M. \& Turnbull, L. 1995. L'accoucheuse traditionnelle dans la protection maternelle et infantile et la planification familiale. Genève : OMS.

\section{NOTES}

1.Cf. Vangeenderhuysen \& Mounkaila (1997 : 6) qui citent un document OMS-UNICEF

2.Attama, S., Seroussi, M., Kourguéni, A.L, Koché, H. \& Barrère, B (1999)

3.Pour l'ensemble du pays y inclus Niamey, les accouchements à domicile représenteraient 81 \% des naissances (Attama, S., Seroussi, M., Kourguéni, A.L, Koché, H. \& Barrère, B., 1999). La même étude rappelle que la fécondité au Niger est la plus élevée au monde (7,5 enfants en moyenne par femme) et qu'une femme sur deux a son premier enfant à 18 ans.

4.Le taux de couverture sanitaire est estimé à $45 \%$ (populations habitant à moins de 5 $\mathrm{km}$ d'un dispensaire), Niamey inclus

5.Cf. Jaffré \& Peual (1993) 
6.Cette étude a été menée sur demande du projet "Maternité sans risques" et du FNUAP à Niamey. Notre équipe de socio-anthropologie de la santé au Niger a successivement travaillé depuis 1993 sur tes représentations et pratiques populaires en matière de santé (cf. les dossiers établis par A. Moumouni, A. Souley et A. Tidjani Alou dans les n ${ }^{\circ}$ 3, 4, 5 et 6 du Bulletin du Programme de recherche"Concepts et conceptions populaires relatifs à la santé, à la souffrance et à la maladie en Afrique de l'Ouest", et les articles de Jaffrè, \& Moumouni 1993 ; Jaffré \& Olivier de Sardan 1996 ; Olivier de Sardan 1994 ; Souley 1999), puis sur les interactions entre populations et services de santé (cf. entre autres enquêtes une étude en cours à Niamey dans trois maternités). Le rapport qui a servi de base au présent article comprend des données sur les représentations et pratiques populaires relatives à la grossesse, et aux soins du nouveau-né qui n'ont pas été retenues ici : cf."Recherche socio-anthropologique sur les complications de la grossesse, de l'accouchement et du post-partum (représentations et pratiques populaires au Niger)", J.P. Olivier de Sardan, A. Moumouni , A. Souley, Rapport final (mai 1997), Niamey, FNUAP (texte publié dans les Working Papers on African Societies, $\mathrm{n}^{\circ}$ 34, Berlin, Das Arabische Buch, 1999). Les citations d'informateurs(trices) du présent article sont renvoyées dans ce rapport à un index nominatif des locuteurs(trices).

7.Manifestement, face à un enquêteur de la ville, il y a, dans le cas ici rapporté, une peur de révéler des comportements non conformes avec la norme officielle, qui est de respecter les consignes des services de santé. Aussi les déclarations sur les 4 voyages de $15 \mathrm{~km}$ pour les $\mathrm{CPN}$, la non-ingestion de toute décoction, la rapidité de l'évacuation, doivent être comprises dans ce contexte...

8.Le sentiment vague qu'il y a communication entre les circuits digestif et reproductif est assez général. L'embryon est censé être "branché" directement sur le circuit digestif pour s'alimenter. D'ailleurs on considère que si la mère ne mange pas un jour, l'enfant va bouger dans son ventre, de faim, jusqu'à ce qu'elle mange et qu'il soit ainsi rassasié. D'autre part, des entités comme weyno ou zahi (cf. note 8) sont censées troubler à la fois les fonctions sexuelles et digestives.

9.Pour ces deux entités nosologiques populaires liées au "ventre", et quelques autres du même type dans d'autres langues, cf. Jamé \& Olivier de Sardan (1999)

10.En référence à cette position, "j'ai trois enfants" pourra se dire en zarma "j'ai trois genoux"(kangey hinza) ou en hausa "j'ai trois agenouillements" (durkushina ukku).

11.Ils portent un nom particulier (par exemple en zarma bargaw, en hausa bakoy) 12. Cette expression est plutôt utilisée en pays songhay ou kado. Rappelons que le songhay et le zarma sont deux parlers très proches d'une même langue.

13.Litt. "cœur" : l'estomac, bien que distingué anatomiquement par un terme particulier (buzugu en zarma et tumbi en hausa) est dans le langage courant appelé "cœur" (bine en zarma, zuciya en hausa).

14.Litt. et par euphémisme "femme en couche"; en hausa on dira "elle est partie avec deux corps", ou "elle était nue quand elle est partie".

15.Cf. Olivier de Sardan 1982 : 207-209.

16.Mais il y a malgré tout une forme d"'intégrisme rampant", non majoritaire loin de là. mais non sans influence, porteur d'un "nouvel ordre moral", qui tout à la fois s'attaque aux guérisseurs traditionnels, aux examens des femmes par des personnels de santé masculins, aux naissances "illégitimes", à la régulation des naissances...

17.Cf. Mangay-Maglacas, A. \& Pizurki, H., 1983, et Verderese, M. \& Tumbull, L.(1995)

18.Cf."Guide de formation des matrones", Ministère de la Santé Publique du Niger (1984) 
19.Notons que, en ces temps de menace de SIDA, non seulement l'usage de gants est inexistant pour les matrones, mais aussi que la nécessité d'une telle protection leur est inconnue.

20."La notion de travail long est bien difficile à préciser, même pour certains professionnels (...) Pour les patientes, le travail commence dès les premières douleurs qui peuvent correspondre au "faux travail "caractérisé par des contractions utérines irrégulières, (...) sans véritable dilatation" (Vangeenderhuysen \& Mounkana 1997 : 53)

\section{AUTEURS}

\section{JEAN-PIERRE OLIVIER DE SARDAN}

Equipe de socio-anthropologie de la santé, IRD, BP 11416, Niamey, Niger ADAMOU MOUMOUNI

Equipe de socio-anthropologie de la santé, IRD, BP 11416, Niamey, Niger ABOUBACAR SOULEY

Equipe de socio-anthropologie de la santé, IRD, BP 11416, Niamey, Niger 